\author{
$5^{\text {th }}$ International Conference \\ on Electrical Engineering \\ ICEENG 2006
}

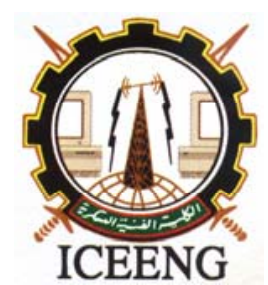

Military Technical College

Kobry Elkobbah, Cairo, Egypt

\title{
ANALYSIS OF THE DESIGN PARAMETERS OF A REMOTE SENSING SATELLITE OPTICAL SENSOR
}

\author{
Fawzy ElTohamy Hasan*PhD., Mahmoud Fathy Mahmoud**Assoc. Prof., \\ Ibrahim Aly Abdel Dayem*PhD., Adel El-Nozahy***PhD., \\ Mohamed El-Sayed Abdel Hady*BSc.
}

\begin{abstract}
:
In this paper, the various parameters that affect the design of the optical sensors used in remote sensing satellites are analyzed. The effects of these design parameters on the spatial resolution of remote sensing satellites are discussed. A simulation of a telescope design is implemented using Zemax package.
\end{abstract}

KEY WORDS: optical sensors, spatial resolution, telescope design, Zemax package.

\section{INTRODUCTION}

The detail discernible in a satellite image is dependent mainly on senor spatial resolution. Spatial resolution of a passive sensor depends on many sensor parameters and satellite orbit altitude. These sensor design parameters, shown in figure (1), include instantaneous field of view, $I F O V$, optics focal length, $f$, entrance aperture diameter, $D$, optics focal number, $F \#$, depth of focus, $\delta$ f, optical system configuration, and detector pixel diameter, $x$, in addition to operating wavelength. The first section of the paper introduces the interdependence of the design parameters on each other and tries to analyze how far these design parameters can be optimized in order to improve the spatial resolution of a remote sensing satellite. The second section of the paper deals with the effect of the design parameters and the integration time, $\mathrm{t}_{\mathrm{int}}$, on the signal-to-noise ratio, $\mathrm{S} / \mathrm{N}$, of an imaging system. The third section introduces a simulation of a telescope design having a Cassegrain configuration implemented by Zemax package.

* Egyptian Armed Forces

** Banha High Technology Institute, Banha, Egypt. 
*** National Laser Institute, Cairo University, Giza, Egypt.

Fig. 1 Optical characteristics of an electro-optical system. 


\section{2- SPATIAL ASPECTS}

The size of the minimum area on the ground viewed by a sensor (determined by multiplying the IFOV by the altitude $H$ of the sensor) is the resolution cell that determines a sensor's maximum spatial resolution. For a feature to be detected, its size generally has to be equal to or larger than the resolution cell. If the feature is smaller than this, it may not be detectable, as the average brightness of all features in that resolution cell will be recorded. It is important to distinguish between pixel size (which was introduced with the use of digital imaging systems) and spatial resolution (ground resolution distance - GRD) - they are not interchangeable. An image pixel represents a certain area on an object (ground sampling distance - GSD), i.e. it is a purely geometric value. Using the objective $G R D$ definition, the smallest object that could be resolved in an electro-optical system would have a width equal to one pixel size GSD and would be separated from a similar object by a distance also equal to one pixel size $G S D$. Therefore, the best $G R D$ that can be achieved with an electro-optical system is twice the $G S D$, i.e., $G R D=2 G S D$ [1]. Sensor design parameters that affect spatial resolution are analyzed in the incoming sub-sections.

\section{Effect of Instantaneous Field of View (IFOV)}

Rayleigh angular resolution, $\theta_{R}$, defines two point sources as just resolved when the peak of one point source image coincides with the first zero of the second point source image [2] (figure 2). Thus, Rayleigh angular resolution defines the IFOV of the sensor. Also, the $I F O V$ may be defined as being the geometric size of the image projected by the detector on the ground through the optical system, called often pixel foot-print [4]. $\theta_{R}$ measured in radian is given by [2],

$$
\theta_{R}=1.22 \frac{\lambda}{D}
$$

with $\lambda$ representing the radiation central wavelength, and $D$ the entrance aperture diameter.

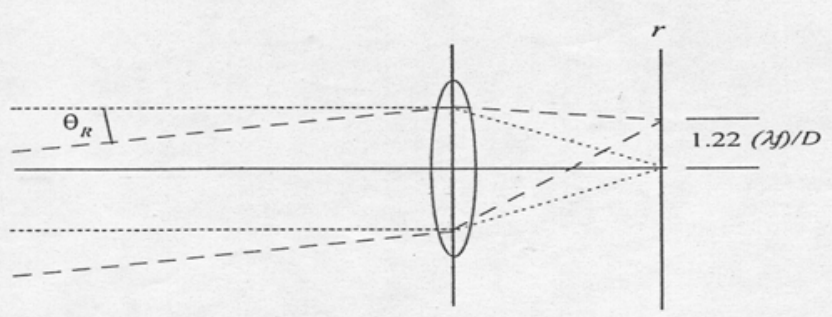




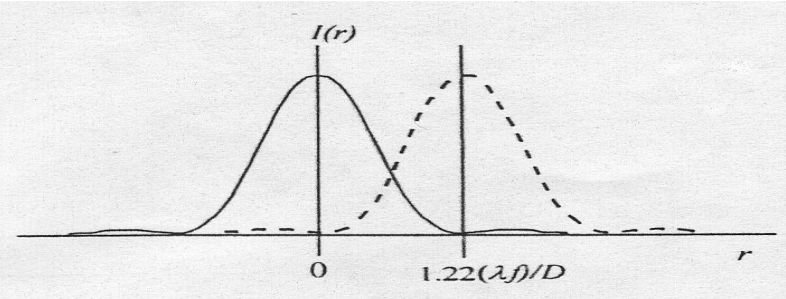

Fig. 2 Rayleigh resolution criterion

For most electro-optical systems, the optical part may be considered near diffractionlimited and in focus [5]. The Airy disc diameter, $d_{\text {Airy }}$, caused by diffraction is one of the important parameters that may be related to the spatial resolution.

$$
d_{\text {Airy }}=2.44 \lambda F \#
$$

with $F \#=f / D$, decreasing $F \#$ of the system will result in low diffraction-limited spot sizes, and thus an enhanced spatial resolution. Also, At small $F \#$, the image brightness will be improved (it is proportional to $\left.1 /(F \#)^{2}\right)$. However, at small $F \#$, the effects of optical aberrations generally become worse due to increasing $\mathrm{D}$, leading to a degraded image quality.

(3)

$$
\begin{aligned}
& G R D=d_{\text {Airy }} H \\
& G R D=2.44 \lambda H / D
\end{aligned}
$$

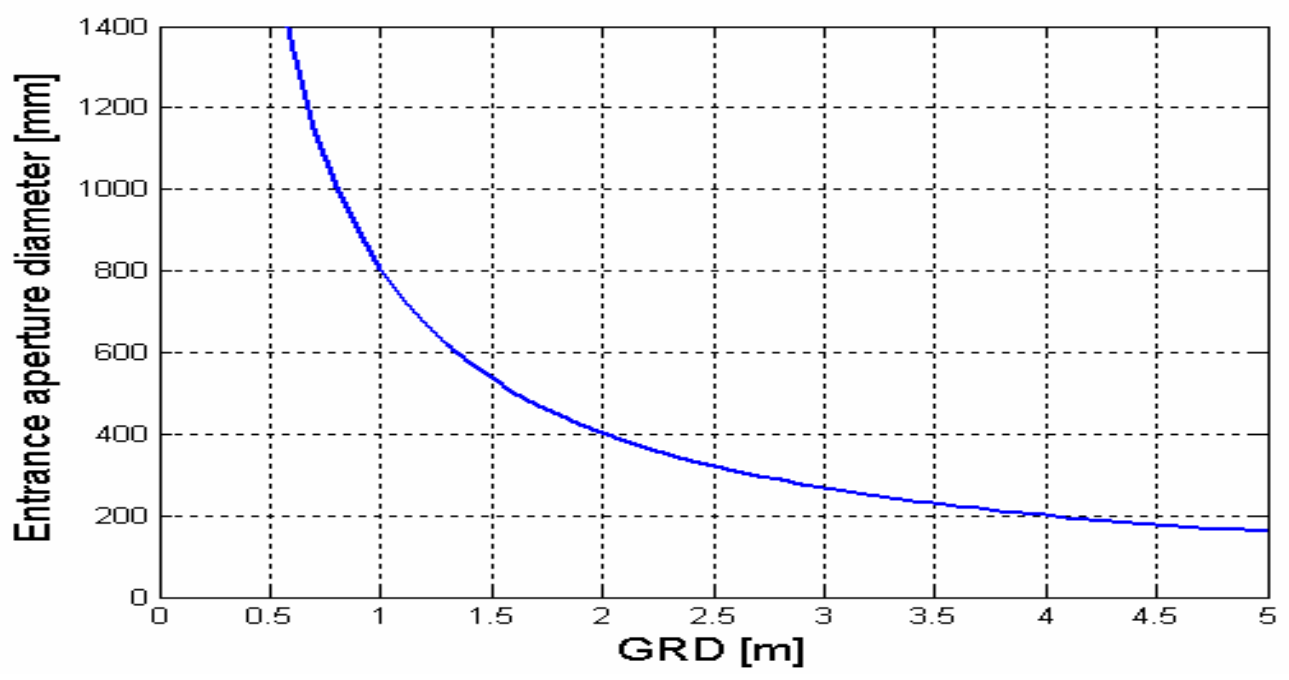


Fig. 3 Dependence between $D$ and $G R D$ for $H=600 \mathrm{Km}$ and $\lambda=0.55 \mu m$.

Figure (3) shows the dependence between the $G R D$ and the designed entrance aperture diameter $D$ using pushbroom imaging technique in the visible band (central wavelength = $0.55 \mu \mathrm{m})$. GRD is improved by minimizing sensor's $I F O V$. At a given wavelength, $I F O V$ could be decreased by increasing $D$, i.e. decreasing $F \#$ of the system, or using a long focal length optics for the same detector pixel diameter, $x$.

\section{Effect of detector pixel diameter (x)}

When the detector pixel diameter: $x \geq d_{\text {Airy }}$, the system is detector limited (diffractionlimited), the spatial resolution is determined by the detector dimension. Otherwise the optics determines the spatial resolution [5]. Figure (4) shows the dependence between $d_{A i r y}$ and $F \#$. The optics designs should be near to the borderline on the optics limited side in order to get maximum radiation energy for the detector [5]. This limitation leads a designer to select quality factor $(Q)$, as shown in figure (5), to have a suitable image quality.

$$
Q=x / d_{\text {Airy }}
$$

This means that the increase of the quality factor $(Q>1)$ improves the spatial resolution, but it will also increase the data rate. Therefore, $Q$ should be a little greater than 1 , (for example: $Q \approx 1.1$ ).

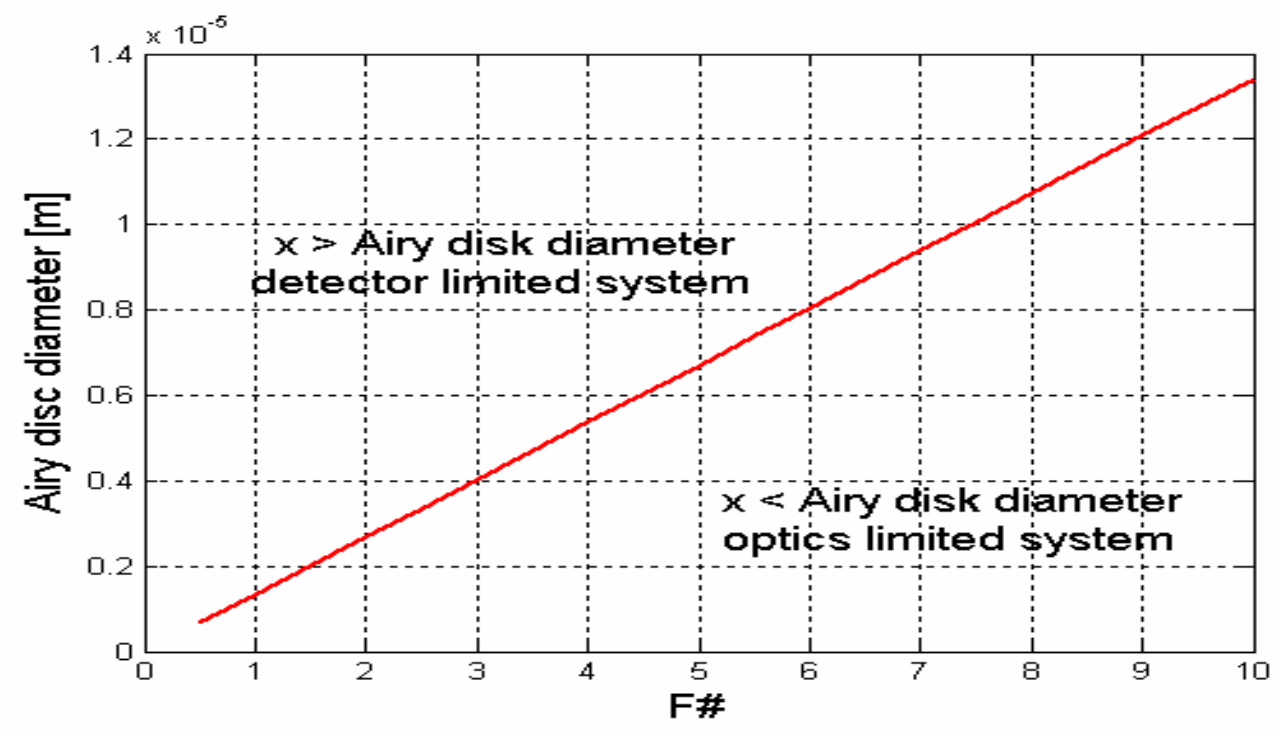

Fig. 4 Dependence between $d_{\text {Airy }}$ and $F \#$ for $\lambda=0.55 \mu m$. 
Fig. 5 Relation between $d_{\text {Airy }}$ and $x$.

As shown in figure (6), the detector pixel diameter $x$ is projected via the optics focal length $f$ to the $G R D$ to be obtained for a given altitude $H$. A relation between $x$ and $G R D$ is given as follows,

(5)

$$
\begin{aligned}
& x / G R D=f / H \\
& G R D=x H / f
\end{aligned}
$$

The smaller the detector element, the shorter the focal length $f$ for the same GRD at given orbit altitude $H$. With smaller detector sizes less energy is integrated.

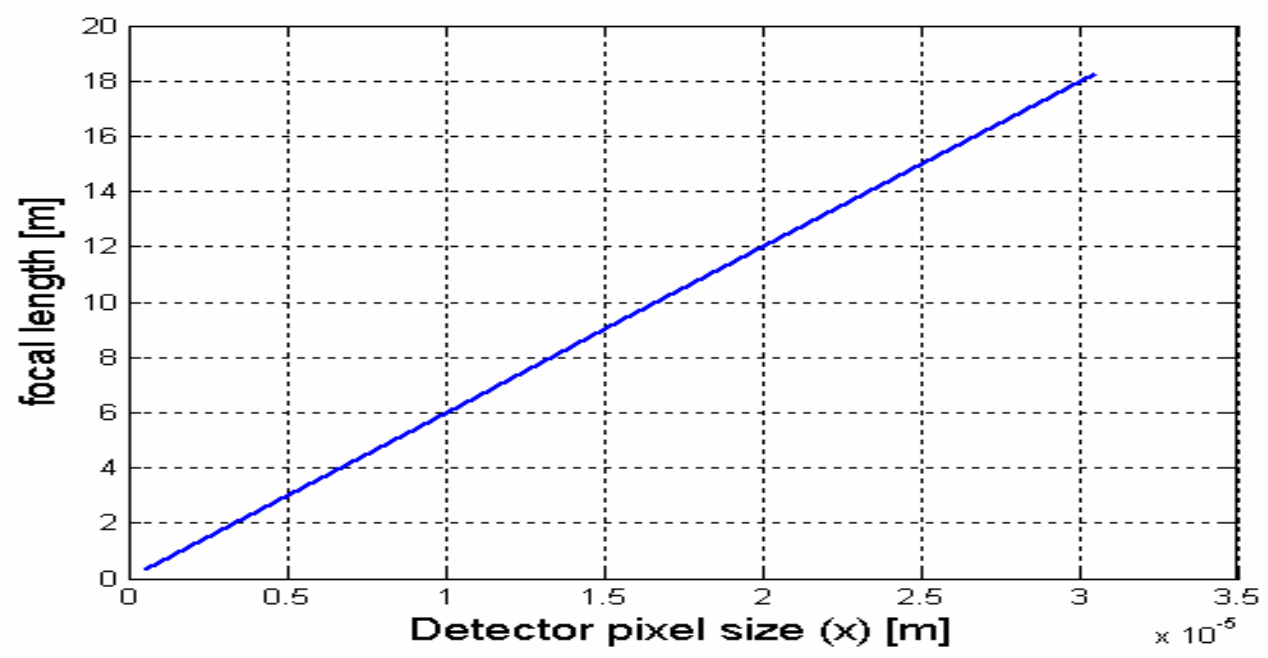

Fig. 6 Dependence between $f$ and $x$ for $G R D=1 \mathrm{~m}$ and $H=600 \mathrm{~km}$.

If the sensitivity of the detector pixel element is not sufficient to obtain the necessary $S / N$, 
then longer integration time is required. This could be achieved by time delay and integration (TDI) imaging technique instead of a simple pushbroom [3].

On the other hand, to improve the spatial resolution for a given $x$ and $H$, this implies increasing the optics focal length as shown in figure (7).

Fig. 7 Relation between $f$ and $\theta_{R}$

\section{Effect of depth of focus $\left(\delta_{\mathrm{f}}\right)$}

Another aspect of the imaging properties of the payload is the depth of focus $(\delta f)$, which means the maximum distance the image plane can be moved away from the exact focus and still have an acceptable focused image, i.e. there is no clear demarcation between being in-focus or out-of-focus.

In figure (8), the rays passing through the edge of the entrance aperture from an on-axis point source are shown. The image plane is moved a distance $\delta f$ leading to the formation of a light spot on the image plane whose radius equals to the Airy disc radius, $r_{o}$.

$$
\delta f \equiv \pm 2 \lambda(F \#)^{2}
$$


Fig. 8 Relation between $F \#$ and $\delta f$.

In the object plane, this expression results in a depth of field $\approx D^{2} / 2 \lambda$. In reality the depth of focus depends on an acceptable image quality, not on any other criteria [3]. From eq.(6) it is clear that the depth of focus is directly proportional to the square of the focal number, i.e. inversely proportional to the square of the entrance aperture diameter $D$.

\section{RADIOMETRIC ASPECTS}

\section{Factors affecting the $\mathrm{S} / \mathrm{N}$ of an imaging system}

The optical system transfers the radiance from the scene under observation to the detector kept at the focal plane (Figure 1). The radiant flux (power), $\phi\left(\theta_{t}\right)$, measured in watt, delivered to the detector element is given by [7],

$$
\phi\left(\theta_{t}\right)=\frac{-}{4} O_{e} \Delta \lambda L_{\lambda}\left(\theta_{R}\right)^{2} D^{2} \cos ^{4} \theta_{t}
$$

in which, $L_{\lambda}$ is the target spectral radiance $\left[\mathrm{W} \mathrm{m}^{-2} \mathrm{sr}^{-1} \mu \mathrm{m}^{-1}\right], \Delta \lambda$ is the spectral bandwidth of the radiation to be measured $[\mu m], O_{e}$ is the optical efficiency - transmittance of the optical system, including atmosphere $\left(O_{e}<1\right)$, and $\theta_{t}$ is the angle of image location with respect to the optical axis.

It can be deduced that the radiant flux impinging the detector surface is not a function of range, the only significant changes in flux from geometric considerations are due to the sensor's entrance aperture diameter and the sensor's $I F O V$.

If the scene is observed for a time ${ }^{t_{\text {int }}}$ [s], then the radiant energy, $Q\left(\theta_{t}\right)$, measured in Joule, received by the detector is given by [7],

$$
Q\left(\theta_{t}\right)=\phi\left(\theta_{t}\right) t_{\mathrm{int}}
$$




$$
Q\left(\theta_{t}\right)=\frac{-}{4} O_{e} \Delta \lambda L_{\lambda}\left(\theta_{R}\right)^{2} D^{2} \cos ^{4} \theta_{t} t_{\mathrm{int}}
$$

For improving high spatial resolution, IFOV has to be reduced. As $I F O V$ is reduced, to get the same energy,

The entrance aperture diameter should be increased, wherein optical size increases, or the spectral bandwidth of the radiation, $\Delta \lambda$, has to be broadened which means the spectral resolution have to be compromised, or the integration time should be increased. Thus one sees a strong inter-dependence amongst various resolution requirements. Since realizing the best of all the resolutions critically influences the complexity of the sensor, a suitable choice for each parameter is essential. Since the signal recorded is proportional to the integration time, the sensor designer tries to maximize the integration time within practical engineering limitations. In fact, maximizing the integration time is an important consideration for the choice of the sensor type [7].

Another important consideration in sensor design is balancing the objectives of keeping the sampled spectral bands narrow, in order to improve the sensor's spectral resolution, while maintaining a high $S / N$. The narrower the wavelength band, the less total radiant energy will be incident upon the detector element. This is because a smaller "slice" of the total radiant flux is being sampled. As a result, the strength of the signal will be reduced relative to the magnitude of the background noise of the detector. In order to maintain high image quality, the $\mathrm{S} / \mathrm{N}$ must be improved by the previously mentioned ways.

\section{Effect of integration time on $\mathrm{S} / \mathrm{N}$}

The number of photoelectrons $\left(n_{p h}\right)$ generated in a solid state camera is given by [8],

$$
n_{p h}=\frac{A_{d} \cdot T_{\text {optics }} \cdot t_{\text {int }}}{4 F \#^{2}} \int_{\lambda_{1}}^{\lambda_{2}} R_{d}(\lambda) \phi(\lambda) d \lambda
$$

in which, $A_{d}$ is the detector area $\left[\mathrm{m}^{2}\right], T_{\text {optics }}$ is the transmission of the optics, $R_{d}$ is the

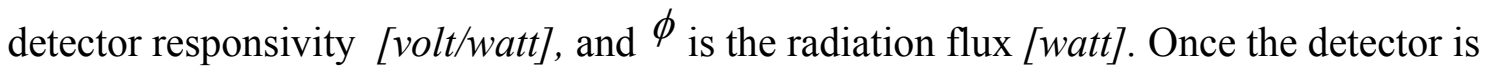

selected, $A_{d}$ and $R_{d}$ are given. $\phi$ is also given as well as $F \#$ and $T_{\text {optics }}$ when the optics is selected or designed taking into account the technological or mission constraints. $\Delta \lambda$ is fixed in most cases, so that the only real variable part is $t_{\text {int }}$.

For a satellite in Low Earth Orbit (LEO), the satellite ground track velocity is about 7 $\mathrm{km} / \mathrm{s}$. In other words, the dwell time is $1 \mathrm{~ms}$ for a GSD of $7 \mathrm{~m}$. For high resolution imagers with $G S D$ of about $1 \mathrm{~m}, t_{\text {int }}<1 / 7 \mathrm{~ms}$ is too short for a sufficient good signal and $\mathrm{S} / \mathrm{N}$. 


$$
t_{\text {int }}(1 \mathrm{~m}) / t_{\text {int }}(10 \mathrm{~m})=1 / 10
$$

Even more severe is the influence of the $I F O V$ :

$$
\operatorname{IFOV}(1 \mathrm{~m}) / \operatorname{IFOV}(10 \mathrm{~m})=1 / 100
$$

Combining both aspects into account, reducing the GSD by a factor of $10^{-1}$ causes a time related and geometry related decrease of energy at the detector of about $10^{-3}$ [8]. Thus to overcome this obstacle, TDI technology with $N$ detector arrays is implied in order to improve the $S / N$ by the factor of $\sqrt{N}$ compared to a single detector array (normal pushbroom imaging), where signal adds in phase but noise in quadrate (this technology is used in the Ikonos and QuickBird missions), or the slow-down mode is used in order to decrease the ground track velocity of the line projection on the ground surface with respect to the satellite velocity in order to obtain the necessary integration time.

\section{TELESCOPE SIMULATION}

The first step in designing a telescope for a high resolution electro-optical sensor is the choice of its configuration which is mainly influenced by the mission limitations and performance requirements put upon the remote sensing satellite. The second step in the design is using a software package for telescope simulation. A typical software packages used in optics simulations include Zemax, Opti-cad, Opal, etc....

In this section, Zemax package is utilized for simulating a Cassegrain telescope for an electro-optical sensor of a remote sensing satellite using pushbroom imaging technique operating at a sun- synchronous orbit and its design parameters are shown in table (1).

\begin{tabular}{|c|c|c|c|c|c|c|c|c|c|}
\hline $\begin{array}{c}\text { Swath } \\
\text { width }\end{array}$ & $\begin{array}{c}\text { Total } \\
\text { FOV }\end{array}$ & F\# & $\begin{array}{c}\text { Entrance } \\
\text { aperture } \\
\text { diameter }\end{array}$ & IFOV & $\begin{array}{c}\text { Effective } \\
\text { focal } \\
\text { length }\end{array}$ & $\begin{array}{c}\text { No. of } \\
\text { detector } \\
\text { pixels }\end{array}$ & $x$ & $G R D$ & $H$ \\
\hline $8192[\mathrm{~m}]$ & $\begin{array}{c}13.653 \\
{[\mu \mathrm{rad}]}\end{array}$ & 5.589 & $40.26[\mathrm{~cm}]$ & $\begin{array}{c}1.6667 \\
{[\mu \mathrm{rad}]}\end{array}$ & $2.25[\mathrm{~m}]$ & 4096 & $7.5[\mu \mathrm{m}]$ & $2[\mathrm{~m}]$ & $600[\mathrm{Km}]$ \\
\hline
\end{tabular}

Table 1. Electro-optical sensor design parameters.

The designed telescope, shown in figure (9), has a Cassegrain configuration with a parabolic primary mirror and a hyperbolic secondary mirror [6]. To project the telescope, simulations with Zemax software is performed to optimize its compactness, a suitable correction for the optical aberrations and a reliable modulation transfer function. The Cassegrain configuration reduces the spherical aberration and the coma, the astigmatism aberration still present in the telescope due to the non-spherical geometry of both mirrors. The field curvature has been corrected by placing a doublet of plano-convex lenses outside the telescope. The first lens will be made up of silica, while the second lens will be made up of BK7. In order to minimize the field curvature the lenses have to fulfill the Petzval condition, $n_{1} f_{1}+n_{2} f_{2}=0[2,6]$, where $\left(n_{1}\right)$ and $\left(n_{2}\right)$ are the refractive indices of the two lenses, and $\left(f_{1}\right)$ and $\left(f_{2}\right)$ are the focal lengths of the two lenses. 


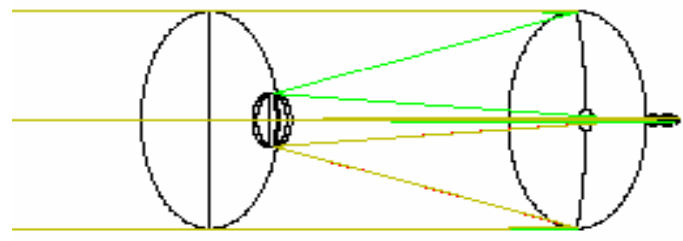

(Figure 9) A Zemax simulation of the Cassegrain telescope with the doublets of lenses to correct field curvature.

The transverse ray fan plot is reported showing variation of ray position at the detectors over a field of angles which simulates the $8192 \mathrm{~m}$ swath seen by the telescope from a 600 Km altitude.

The primary mirror has a diameter of $40 \mathrm{~cm}$ and the secondary mirror has a diameter of $9.9 \mathrm{~cm}$. The telescope focal length is $225 \mathrm{~cm}$. In table (2) all the telescope parameters are recorded.

\begin{tabular}{|c|c|c|c|c|c|c|}
\hline $\begin{array}{c}\text { Working } \\
\text { F\# }\end{array}$ & $\begin{array}{c}\text { Distance } \\
\text { Primary- } \\
\text { Secondary }\end{array}$ & $\begin{array}{c}\text { Sec. mirror } \\
\text { conic coeff. }\end{array}$ & $\begin{array}{c}\text { Pr. mirror } \\
\text { conic coeff. }\end{array}$ & $\begin{array}{c}\text { Secondary } \\
\text { mirror } \\
\text { focal length }\end{array}$ & $\begin{array}{c}\text { Primary } \\
\text { mirror } \\
\text { focal length }\end{array}$ & $\begin{array}{c}\text { Telescope } \\
\text { Focal length }\end{array}$ \\
\hline 5.5886 & $52 \mathrm{~cm}$ & -2.8657 & -1 & $23 \mathrm{~cm}$ & $69 \mathrm{~cm}$ & $225 \mathrm{~cm}$ \\
\hline
\end{tabular}

Table 2. optical telescope parameters

\section{CONCLUSION}

The constraints of building a remote sensing satellite, with limited volume and weight, and an enhanced image quality have resulted in innovative approaches to the design of the telescope as an essential part of an electro-optical system, these design parameters should be optimized. Also, a trade-off of the geometric design parameters of an optical sensor, and their influence on the various performance parameters is taken into account as well as the radiometric aspects concerning them.

\section{REFERENCES}

Theobald, E. Glen, "When Is a Meter a Meter", Imaging Notes, vol.13 no.2, September/ October, 1998.

Smith, Warren J., “Modern Optical Engineering”, Second edition, 1990.

Larson, Wiley J., and Wertz, James R., "Space Mission Analysis and Design", Second edition, 1992.

Joseph, George, "How well do we understand Earth observation electro-optical sensor parameters?”, ISPRS Journal of Photogrammetry and Remote Sensing 55 (2000) 9-12. 
Center, Optical Information Systems.

ZEMAX, Optical design program User's Guide, Version 7.0, 1998.

K. Kasturirangan, "Science and Technology of Imaging from Space", Current Science, vol.

87, no.5, 10 September 2004.

Pica, Giulia, "High Resolution Deployable Telescope for Satellite Application",

Proceedings of Spie's Remote Sensing, Europe 2003. 